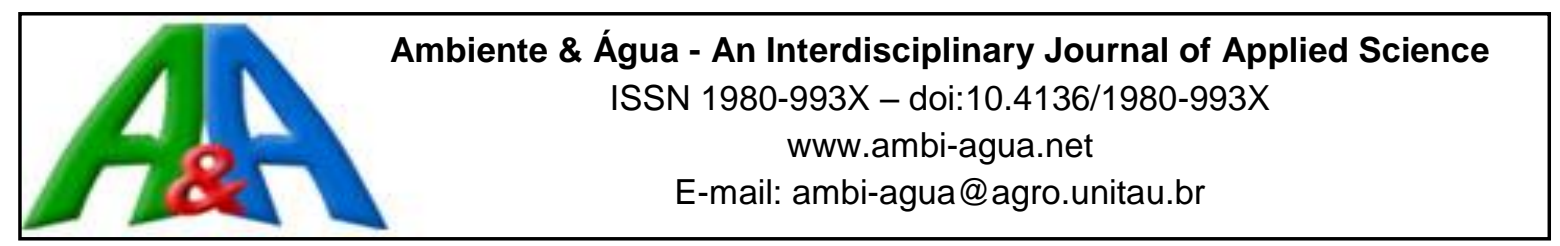

\title{
Aplicación de modelos unidimensionales de flujo en suelos no saturados y transporte de herbicidas en zonas agrícolas
}

\author{
doi: 10.4136/ambi-agua.1165 \\ Received: 05 Sep. 2013; Accepted: 21 Jul. 2014 \\ Teresa Maria Reyna*; Jorge Armando Linares; \\ Santiago Reyna; María Lábaque \\ Universidad Nacional Córdoba, Córdoba, Argentina \\ Facultad de Ciencias Exactas, Físicas y Naturales \\ *Autor correspondiente: e-mail: teresamaria.reyna@gmail.com, \\ linjorge@gmail.com, santiagoreyna@gmail.com, mlabaque@gmail.com
}

\section{RESUMEN}

La predicción del comportamiento de los plaguicidas liberados en el ambiente es necesaria para anticipar y minimizar, impactos adversos fuera del punto de aplicación. Esto significa que debemos comprender qué le sucede a un pesticida que ha sido aplicado en el campo, y predecir su destino en el ambiente. Con este objetivo se estudió una parcela experimental del Instituto Nacional de Tecnología Agropecuaria (INTA) en la localidad de Manfredi (Córdoba; Argentina). Se realizaron mediciones del contenido de agua en suelo (por sonda de neutrones y por el método gravimétrico) y la precipitación ocurrida en el sitio durante un período de tiempo con el fin de modelar el proceso de humedecimiento. También se midieron los contaminantes a distintas profundidades y a distintos tiempos de aplicación de atrazina al predio. Con estos datos se buscó modelar el proceso con el programa HYDRUS. Los resultados obtenidos muestran que el modelo subestima los contenidos de humedad presentes en el horizonte superior, mientras que para profundidades mayores frente a eventos de precipitaciones normales y de riego, el frente de humedecimiento no genera una recarga directa de la freática. Los resultados obtenidos muestran que la simulación numérica resulta una herramienta válida para el estudio del movimiento del flujo y el transporte de sustancias contaminantes en la zona no saturada. La principal limitante para evaluar la validez de estos modelos son la poca disponibilidad de datos de estudios de campo.

Palabras claves: humedad en suelos, transporte de contaminantes, agroquímicos.

\section{Aplicação de modelos de coluna de fluxo em solos não saturados e transporte de herbicidas em áreas agrícolas}

\section{RESUMO}

É necessário se antecipar a previsão do comportamento de pesticidas liberados no meio ambiente para se minimizar os impactos negativos fora do ponto de aplicação. Isso significa que se tem que entender o que acontece com um pesticida que foi aplicado no campo e prever o seu destino no meio ambiente. Dessa forma, uma parcela experimental do Instituto Nacional de Tecnologia Agropecuária (INTA) na cidade de Manfredi (Córdoba, Argentina) foi 
estudada. Foram realizadas medições do conteúdo de água no solo (por tubo de neutrons e pelo método da gravidade) e da precipitação no local, a fim de modelar o processo de humedecimento. Poluentes também foram medidos em diferentes profundidades e em diferentes épocas de aplicação da atrazina no local. Com esses dados modelou-se o processo utilizando-se o programa HYDRUS. Os resultados mostram que o modelo subestima o teor de humidade presente no horizonte superior, enquanto que para profundidades maiores em condições de eventos de chuvas normais e irrigação, a frente de umedecimento não ocasiona recarga direta de água do freático. Os resultados mostram que a simulação numérica é uma ferramenta válida para o estudo da circulação do fluxo de transporte e de contaminantes na zona insaturada. A principal restrição para avaliar a validade destes modelos é a limitada disponibilidade de dados de estudos de campo.

Palavras-chave: umidade do solo, transporte de contaminantes, agroquímicos.

\title{
Application of a one-dimensional model of flow in unsaturated soils and transport of herbicides in agricultural areas
}

\begin{abstract}
In order to minimize adverse impacts beyond the place of application, it is necessary to predict the behavior of pesticides released into the environment. This means that we must understand what happens to a pesticide that has been applied in the field and that we must be able to predict its destination in the environment. For this purpose, we studied an experimental agricultural plot at the National Institute of Agricultural Technology (INTA) in the town of Manfredi (Córdoba, Argentina). The contents of water in the soil were measured (by neutron probe and by the gravimetric method) along with precipitation at the site during a certain period in order to model the process of humidification. Pollutants' contents were also measured at various depths and at different times of atrazine application. This data was then used to model the process using the software HYDRUS. The results show that the model underestimates the moisture content present in the upper horizon, while in deeper layers, under normal events of rainfall and irrigation, the humidification front does not generate a direct recharge from the phreatic water. The study established that numerical simulation is a valid tool for studying the movement of flow and the transport of contaminants in the unsaturated zone. The lack of field data is main limitation to assessing the validity of the model.
\end{abstract}

Keywords: moisture in soil, contaminant transport, agrochemicals.

\section{INTRODUCCIÓN}

Desde el inicio del movimiento agrícola conocido como revolución verde, a principios de la Segunda Guerra Mundial, se ha podido presenciar el desarrollo de una agricultura que más que convencional, como se suele denominar, se podría llamar industrializada. Esto último responde fundamentalmente al empleo de abonos químicos y pesticidas, a la producción de nuevas variedades de aspecto más atractivo y a la progresiva mecanización de la forma de cultivo más practicada: el monocultivo. En las primeras fases del desarrollo de la agricultura convencional o industrializada se observaron incrementos mundiales registrados de las cosechas de cereales, leguminosas, oleaginosas, fibras y raíces, para los períodos 1929-1979. Posteriormente, se ha ido produciendo una disminución de los incrementos de la productividad, especialmente en aquellos países que más tempranamente adoptaron las técnicas de la revolución verde (Romera, 2010). 
Con respecto a los plaguicidas la mayoría son compuestos que no se encuentran naturalmente en el ambiente y por lo tanto, concentraciones detectables indican contaminación (Chapman, 1992).

Los suelos son considerados descontaminantes naturales, debido a su heterogeneidad, tanto por su composición como por la diversidad de procesos que ocurren. El conjunto de propiedades físicas, químicas, y biológicas que posee hacen que actúe como una barrera protectora de otros medios, especialmente de las aguas superficiales y subterráneas. Por lo que la hipótesis ampliamente aceptada de que la fracción no saturada del suelo constituye una defensa efectiva contra la penetración de plaguicidas es por lo tanto cuestionable (Giuliano, 1995).

La predicción del comportamiento de los plaguicidas liberados en el ambiente es necesaria para anticipar, y por ende minimizar, impactos adversos fuera del punto de aplicación (Wagenet y Rao, 1990.). Esto significa que debemos comprender qué le sucede a un pesticida que ha sido aplicado en el campo, y predecir su destino en el ambiente. Utilizando esta información, pueden estimarse los probables impactos adversos sobre el agua superficial o subterránea y/o sobre la salud humana. Existen modelos con distinto nivel de complejidad para una variedad de aplicaciones (Bedmar et al., 2004).

Sin duda, la escasez de conocimientos sobre contenidos de plaguicidas en suelos y en los cuerpos de agua luego de su aplicación es debida a la extraordinaria dificultad para la identificación y cuantificación de estos compuestos y de los productos de degradación originados a lo largo de su tránsito por la zona no saturada (Reyna et al., 2013) .

En los últimos años se ha comenzado a prestar atención a los procesos de contaminación de aguas subterráneas ligados a la utilización de fertilizantes, particularmente los nitratos, en las prácticas agrícolas. Se tiene información relativa de su presencia en los acuíferos pero no se conocen acabadamente los mecanismos de migración e interacción en el medio no saturado. Esta carencia es más patente en el caso de los plaguicidas, sustancias químicamente más complejas, implicadas en procesos de transformación y degradación mal conocidos.

En particular para la Física de Suelos la predicción del movimiento del agua y los solutos se hace más compleja en la medida en que la modelación del sistema agua-solutos-sueloplanta sea más cercana a la realidad, es por ello que para lograr establecer elementos de análisis, en general, es preciso simplificar al máximo el modelo del sistema físico y describir sus interacciones y parámetros de la manera más simple posible.

\subsection{Herbicidas}

Los herbicidas son productos destinados para el control de malezas de determinado cultivo no deseadas por su impacto negativo en la producción y rendimientos, estos se encuentran dentro del grupo de productos fitosanitarios los cuales por definición de la Organización Mundial de la Salud (OMS) son todas aquellas sustancias o mezcla de sustancias, destinadas a prevenir la acción de controlar o destruir directamente malezas, insectos, hongos, ácaros, moluscos, bacterias, roedores y otras formas de vida animal o vegetal que puedan resultar perjudiciales tanto para la Salud Pública como para la Agricultura.

Por lo general todos los herbicidas que pertenecen a un mismo grupo genérico actúan de la misma manera, por ejemplo: al conocerse el efecto de la atrazina sobre una maleza (la mayoría de las malezas latifoliadas anuales y algunas gramíneas, como cola de zorro y pasto colorado) también se sabe cómo actúa la simazina, la ametrina y la prometrina, ya que todos estos productos perteneces a la misma familia de las triazinas (Doll, 1982). No existe una única clasificación de herbicidas, ya que los mismos pueden ser agrupados según su naturaleza química, su mecanismo de acción, el momento de aplicación, etc. Cabe aclarar también que un mismo herbicida, puede ser englobado en diversas categorías de clasificación. 
La movilidad de los plaguicidas depende de sus propiedades físicas y químicas en el medio, tales como volatilidad, solubilidad, persistencia y la adsorción en el suelo. La concentración de herbicida en el suelo a lo largo del tiempo - persistencia o residualidad depende de las características propias de la molécula (acidez o alcalinidad de la molécula, solubilidad en agua, presión de vapor) y su interacción con las características del suelo (composición de la fracción arcilla, pH, capacidad de intercambio catiónico, área superficial, contenido de materia orgánica) y de los factores ambientales (temperatura, humedad), conformando una serie de procesos de disipación: volatilización, descomposición fotoquímica o química, escurrimiento superficial, degradación química, descomposición microbiana, lixiviación y adsorción (Díaz Trujillo, 2007).

Si bien existen propiedades específicas que determinan la movilidad de un herbicida en el suelo Goss (1992) y Hornsby (1992) demostraron que el coeficiente de partición en carbono orgánico $\left(\mathrm{K}_{\mathrm{oc}}\right)$ y la vida media de los plaguicidas $\left(\mathrm{T}_{1 / 2}\right)$ pueden utilizarse para comparar sus potenciales de lixiviar a través de la matriz del suelo. Estableciendo que la materia orgánica del suelo es la característica edáfica que más influye sobre el movimiento de los herbicidas. La presencia de capas con materia orgánica produce "atenuación" del flujo del herbicida protegiendo al acuífero de la contaminación.

Por lo tanto para la determinación de la capacidad de infiltración de un herbicida es necesario tener en cuenta: 1) profundidad del acuífero saturado combinado con la dirección predominante del flujo de agua, 2) permeabilidad de los estratos geológicos y de suelo, 3) contenido de materia orgánica del suelo, y 4) $\mathrm{K}_{\mathrm{oc}} \mathrm{y} \mathrm{T}_{1 / 2}$ del plaguicida (Bedmar et al., 2004).

\section{MATERIALES Y MÉTODOS}

Unas de las principales dificultades que se encuentran para modelar los procesos de infiltración es conocer los parámetros de los suelos para lograr una adecuada representación de la realidad. Dado el problema de la heterogeneidad presente en el medio poroso la modelación del sistema agua-solutos-suelo-planta es más complicado. Por lo tanto obtener los parámetros que permiten ajustar el modelo lo más cercano a la realidad es lo que lleva la mayor parte de tiempo y esfuerzo.

Con el objetivo de obtener datos sobre la humedad del suelo y la presencia de contaminantes durante un período de tiempo, se realizaron estudios en una parcela experimental del Instituto Nacional de Tecnología Agropecuaria (INTA) de un área aproximada de 25 ha, que posee un sistema de riego suplementario en funcionamiento tipo pivote central. Durante el año se realiza la rotación de cultivo entre el maíz, soja - trigo y maíz, trigo-soja.

La parcela de análisis se ubica en las coordenadas geográficas $63^{\circ} 44^{\prime} 44.91^{\prime \prime}$ 'Oeste; $31^{\circ}$ 52' 19.08' Sur, Ruta Nacional N9 km 636, Manfredi, Córdoba, Argentina, con una elevación de 295 msnm (Figura 1).

En esta parcela se registraron de forma continua los eventos de precipitación y ausencia de esta. Se registraron también los valores de humedad experimentados por el suelo a distintas profundidades.

La precipitación $(\mathrm{P})$ se ha obtenido a partir de un pluviómetro automático de la estación meteorológica ubicada en el lote de estudio para los meses de julio 2010 hasta mayo del 2011; mientras que la evapotranspiración (ET_0) ha sido calculada según la ecuación combinada de Penman-Monteith (Monteith, 1981), a partir de las variables meteorológicas medidas por la misma estación.

La medición adecuada del contenido de agua en suelo resulta crítica para la estimación de los balances de agua y energía, así como para comprender los procesos biológicos y químicos en todo el sistema suelo-planta (Robinson et al., 2003 y Vereecken et al., 2008). 
Dada la importancia de este parámetro, se trabajó con varios métodos de medición de la humedad en suelos: métodos gravimétricos, sensores de capacitancia y sonda de neutrones. Se analizaron los resultados, ventajas y desventajas de utilizar cada uno.

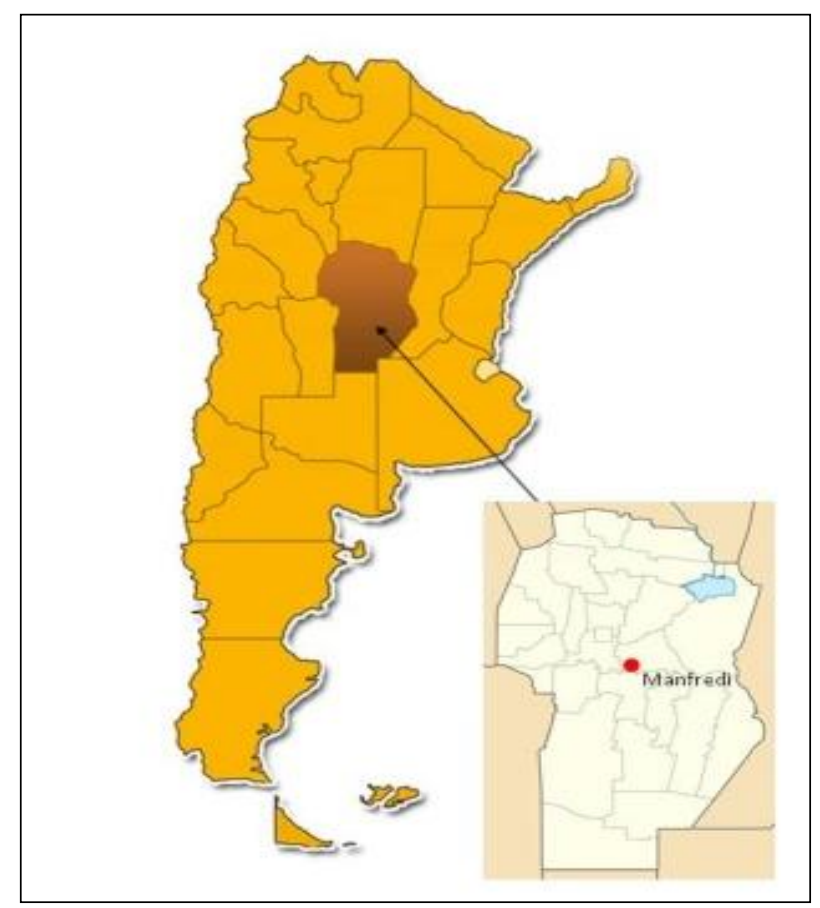

Figura 1. Ubicación de la Zona de estudio en Manfredi, Córdoba, Argentina.

Con respecto a las mediciones de los contaminantes en suelos se tomaron muestras bajo condiciones de labranza cero. La prueba se llevó a cabo bajo el sistema de riego centralizado o pivote central, lo que aseguró el riego uniforme sobre los cultivos.

Durante el desarrollo del experimento se hicieron 6 muestreos de tipo simple aleatorio sistemático y en profundidad: a los 5 , a los 40 y a los $90 \mathrm{~cm}$ con barreno manual (IRAM 29481-1, 2 y 4 y ASTM Standards on Environmental Sampling, 1995). Las muestras se tomaron previo a la aplicación de la Atrazina (concentración de 2 lts/Ha) a los 7, 15, 30, 60, 90 días de dicha aplicación. Por otra parte se determinaron las características edafológicas del suelo estudiado. El suelo del lote sobre el que se realizaron los ensayos fue caracterizado en sus propiedades físicas y químicas en laboratorio, Fósforo Extractable por el método Bray y Kurtz (1945), Nitrógeno de Nitratos (método Fenildisulfónico), Nitrógeno Total por el método Kjeldahl (1883), Materia Orgánica por el método Walkley y Black (1934), Cond. Eléctrica (método relación suelo/agua: 2,5 x 5).

Para la determinación de Atrazina se utilizó un cromatógrafo líquido (HPLC) Alliance 2695 (WATERS), con un detector de espectrometría de masas triple cuádruplo (qQq) Quattro Ultima Pt (Micromass). Las muestras fueron analizadas por Electrospray a presión atmosférica en modo positivo y negativo (API ESI +/-). Se utilizó una columna C18 de 100x2.1 mm Hypersil Gold (Thermo). Un sistema de gradiente entre agua (20 mM de formiato de amonio) y metanol. Las muestras fueron extraídas con una mezcla de agua/acetonitrilo y acido fórmico e inyectadas en el HPLC/MS/MS. Se analizaron por monitoreo de reacción múltiple (MRM) utilizando los siguientes iones específicos: Plaguicida Ion padre Iones hijos Atrazina 216174 (ion de cuantificación) 96 (ion de confirmación). Los 
patrones usados fueron Atrazina Supelco (49085). Las cuantificaciones se hicieron por el método de estándar externo y las curvas fueron preparadas en extracto de matriz.

Con los datos obtenidos se buscó modelar matemáticamente el flujo de agua en suelos no saturados y el transporte de agroquímicos siguiendo el comportamiento de la parcela a lo largo de un período con y sin precipitación y luego de la aplicación de agroquímicos.

Para las modelaciones computacionales se seleccionó el programa HYDRUS (Šimunek et al., 2012). HYDRUS es un paquete de software para simular el movimiento del agua, el calor y solutos en una, dos y tres dimensiones, en medios saturados y no saturados. El paquete consiste en un programa informático de cálculo y una interfaz de usuario basada en gráficos interactivos. La selección del programa se debe a su gran uso a nivel de investigación y ademas de contar con varias actualizaciones que incorporan nuevos módulos como el de modelación inversa, la modelación de contaminantes y modelación con histérisis entro otros.

\subsection{Caracterización del suelo}

La estación experimental del INTA Manfredi está caracterizada dentro de la llanura central Cordobesa, en un área de transición entre la subregión que se conoce con el nombre de plataforma basculada o Pampa alta y la subregión denominada Pampa plana.

El tipo de suelo presente en el lote se caracteriza como un haplustol étnico, limosa gruesa, mixta, térmica para capacidad de uso IIIc. Este suelo presenta característica bien o algo excesivamente drenado, desarrollados sobre los materiales franco limosos que ocupan las lomas más extendidas, casi planas (Inta Manfredi, 2010).

- Horizonte superficial A con una profundidad de $23 \mathrm{~cm}$, se caracteriza por un color en húmedo pardo grisáceo (10YR3/2) y de textura franco limosa, estructura en bloques subangulares medios moderados; friable en húmedo; no plástico; no adhesivo; con presencia de abúndate materia orgánica. El límite inferior es abrupto, suave.

- Horizonte transicional AC con un espesor de $30 \mathrm{~cm}$ se caracteriza por un color en húmedo pardo oscuro a pardo amarillento oscuro (10YR3/3.5) y de textura franco limosa, estructura en bloques subangulares medio débiles a masivo; friable en húmedo; no plástico; no adhesivo; El límite inferior es abrupto, suave.

- Horizonte Ck se encuentra a partir de los $53 \mathrm{~cm}$ de profundidad, se caracteriza por un color en húmedo pardo (7.5YR4.5/4) el tipo de suelo presente es franco limoso, masivo, muy friable en húmedo; no plástico; no adhesivo y fuerte reacción al ácido clorhídrico en la masa del suelo, por lo que se presenta abundante material calcáreo pulverulento diseminado en la masa del suelo.

El tipo de suelo presente dentro del lote se caracteriza como un haplustol étnico, limosa gruesa, mixta, térmica para capacidad de uso IIIc. Este suelo presenta característica bien o algo excesivamente drenado, desarrollados sobre los materiales franco limosos que ocupan las lomas más extendidas, casi planas (Inta Manfredi, 2010.). En la Tabla 1, se resumen las principales características del suelo presentes en la zona de estudio del lote de Manfredi.

Tabla 1. Principales características hidráulicas del suelo.

\begin{tabular}{ccccccccc}
\hline Horizonte & $\begin{array}{c}\text { Profundidad } \\
(\mathrm{cm})\end{array}$ & $\begin{array}{c}\text { Arena } \\
(\%)\end{array}$ & $\begin{array}{c}\text { Limo } \\
(\%)\end{array}$ & $\begin{array}{c}\text { Arcilla } \\
(\%)\end{array}$ & $\begin{array}{c}\text { H.E } \\
(\%)\end{array}$ & $\begin{array}{c}\boldsymbol{\rho}^{*} \\
\left(\mathrm{~g} / \mathrm{cm}^{3}\right)\end{array}$ & $\begin{array}{c}\mathbf{C C}^{* *} \\
\left(\mathrm{~cm}^{3} / \mathrm{cm}^{3}\right)\end{array}$ & $\begin{array}{c}\mathbf{P M P} * * * \\
\left(\mathrm{~cm}^{3} / \mathrm{cm}^{3}\right)\end{array}$ \\
\hline A & $0-23$ & 16.5 & 68.7 & 15.7 & 0,28 & 1,33 & 0,34 & 0,15 \\
AC & $23-53$ & 16.1 & 71.1 & 12.2 & 0,25 & 1,29 & 0,31 & 0,14 \\
C & $53-+$ & 16 & 71.9 & 11.2 & 0,23 & 1,19 & 0,26 & 0,12 \\
\hline
\end{tabular}

* Densidad del suelo;** Capacidad de campo; *** Punto de marchitez permanente. 


\subsection{Condiciones climáticas}

La estación experimental Manfredi se encuentra ubicada entre áreas que por sus deficiencias hídricas la ubican dentro de la región semiárida. La información del registro pluviométrico se obtuvo por medio de una estación meteorológica, ubicada dentro la zona de estudio caracterizando el régimen térmico dentro de la zona como templado con una temperatura media anual de $16.8^{\circ} \mathrm{C}$, el mes más caluroso es enero con una temperatura media de $23.4{ }^{\circ} \mathrm{C}$ y julio corresponde al mes más frío del año con una temperatura media de $9.6^{\circ} \mathrm{C}$, por lo tanto la amplitud térmica anual resulta ser de $13.8^{\circ} \mathrm{C}$ (Inta Manfredi, 2010).

El régimen pluviométrico se asemeja al monzónico, prevalente en el noroeste de Argentina. La suma total media anual de las precipitaciones es de $758 \mathrm{~mm}$ de los cuales un $80 \%$ se concentra durante el semestre de Octubre - Marzo; los meses más lluviosos son Diciembre - Enero, y los más secos son Junio, Julio y Agosto.

\section{RESULTADOS Y DISCUSIÓN}

Utilizando Hydrus, se simuló la variación del contenido de humedad. En el proceso de calibración de los parámetros de van Genuchten-Mualem (VG-M) no se tuvo en cuenta la absorción por las raíces, ya que en el periodo comprendido el suelo se encontraba en descanso, mientras que en la simulación para la obtención de los coeficientes de transporte de la atrazina, se ha adoptado la función para la distribución de las raíces tipo Trapezoidal de Absorción de Gardner (1967), y para la simulación del efecto del estrés hídrico del suelo sobre la absorción radicular se ha utilizado la función de Feddes (Feddes et al., 1978) con los parámetros correspondientes para el cultivo de maíz.

Para el proceso de modelación inversa se tuvieron en cuenta los valores aportados por una campaña de medición, los cuales obtuvieron valores de humedad del terreno aplicando un método de obtención por gravimetría y otro por medio de una sonda de neutrones. Los parámetros del modelo que se obtuvieron optimizados para los datos de la sonda de neutrones se presenta en la Tabla 2.

Tabla 2. Parámetros de vG-M optimizados para los datos de la sonda de nêutrones.

\begin{tabular}{ccccccc}
\hline Horizonte & $\theta_{r} \mathrm{~cm}^{3} / \mathrm{cm}^{3}$ & $\theta_{S} \mathrm{~cm}^{3} / \mathrm{cm}^{3}$ & $\alpha$ & $\mathrm{n}$ & $K_{S} \mathrm{~cm} /$ día & 1 \\
\hline $\mathrm{A}$ & 0,05392 & 0,4402 & 0,008352 & 1,139 & 41,74 & 0,5 \\
$\mathrm{AC}$ & 0,04796 & 0,5073 & 0,001869 & 1,289 & 115,4 & 0,5 \\
$\mathrm{C}$ & 0,04351 & 0,5449 & 0,002747 & 1,3888 & 74,04 & 0,5 \\
\hline
\end{tabular}

Los resultados se presentan en las Figura 2 para la profundidad de $10 \mathrm{~cm}$ u horizonte A. Se observó que la evolución en el tiempo de las medidas experimentales y de los valores simulados siguió una misma pauta, con pequeñas diferencias entre ellos ajustándose mejor a los datos de la sonda de neutrones.

En cuanto a la evolución de los contenidos de agua observados y simulados a 40 y $90 \mathrm{~cm}$ de profundidad muestran un comportamiento muy distinto, observándose un incremento en el contenido de agua de simulación desde el día 65 debido a la lluvia de $1.52 \mathrm{~cm}$ ocurrida el día 22 de Septiembre del 2010. La figura 3 muestra a la profundidad de $90 \mathrm{~cm}$ la evolución en el tiempo de las medidas experimentales y de los valores simulados.

De las modelaciones realizadas y contrastadas con los datos obtenidos de campo se puede observar que los parámetros de la ecuación de VG-M correspondientes a los horizontes AC y $\mathrm{C}$, ajustados mediante modelación inversa no han proporcionado buenos resultados. 
Algunas de las causas se debe a que sólo se dispuso de medidas experimentales del contenido de agua para realizar la calibración, mientras que si se dispone de mediciones de valores de contenido y potencial esto mejoraría los resultados de la calibración (Arbat et al., 2005).
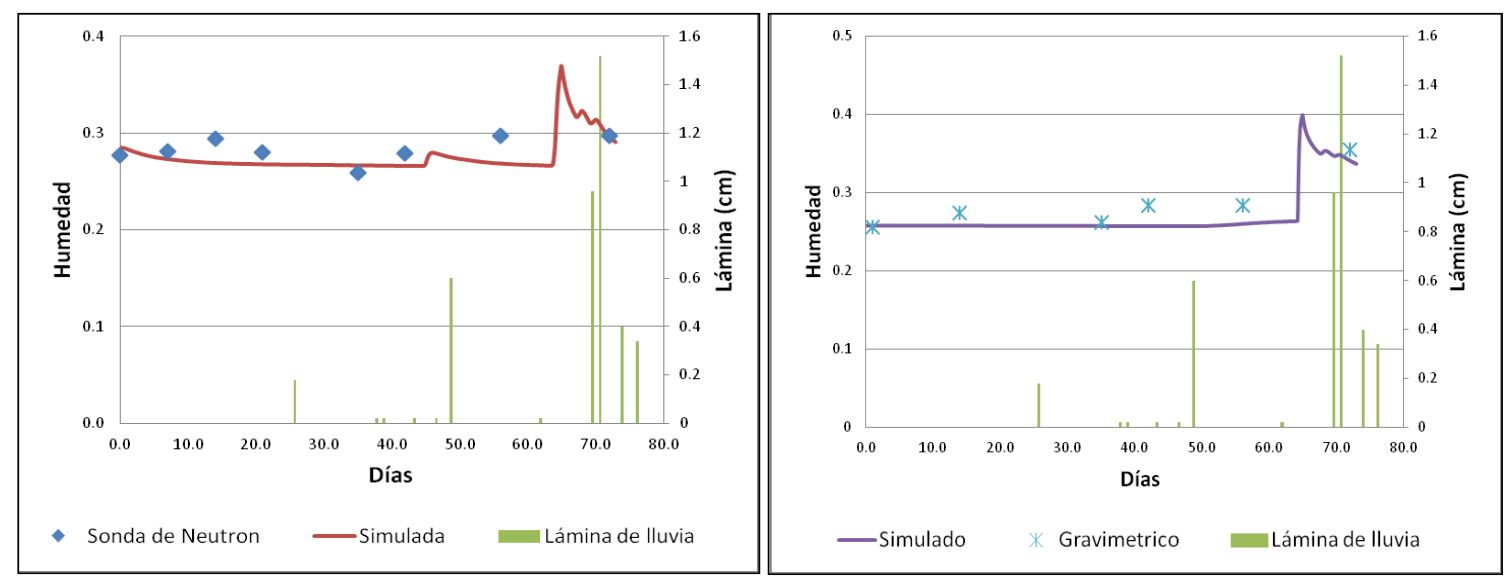

Figuras 2. Resultados de la modelación con Hydrus a profundidad $10 \mathrm{~cm}$. Derecha: Simulación con el modelo ajustado para la sonda de neutrones. Izquierda: Simulación para el modelo ajustado con los datos del método gravimétrico.

Para la obtención de los parámetros optimizados de la ecuación de transporte de la atrazina se plantearon dos escenarios, uno de ellos fue tomando los parámetros del suelo obtenidos a partir de la modelación inversa con los datos observados del sensor de neutrones y el segundo escenario fue considerando los parámetros obtenidos del método gravimétrico. Tanto los datos de condición inicial, de borde y meteorológicos son ajustados para las fechas del 21 de diciembre del 2010 al 25 de mayo del 2011, obteniéndose los siguientes resultados.

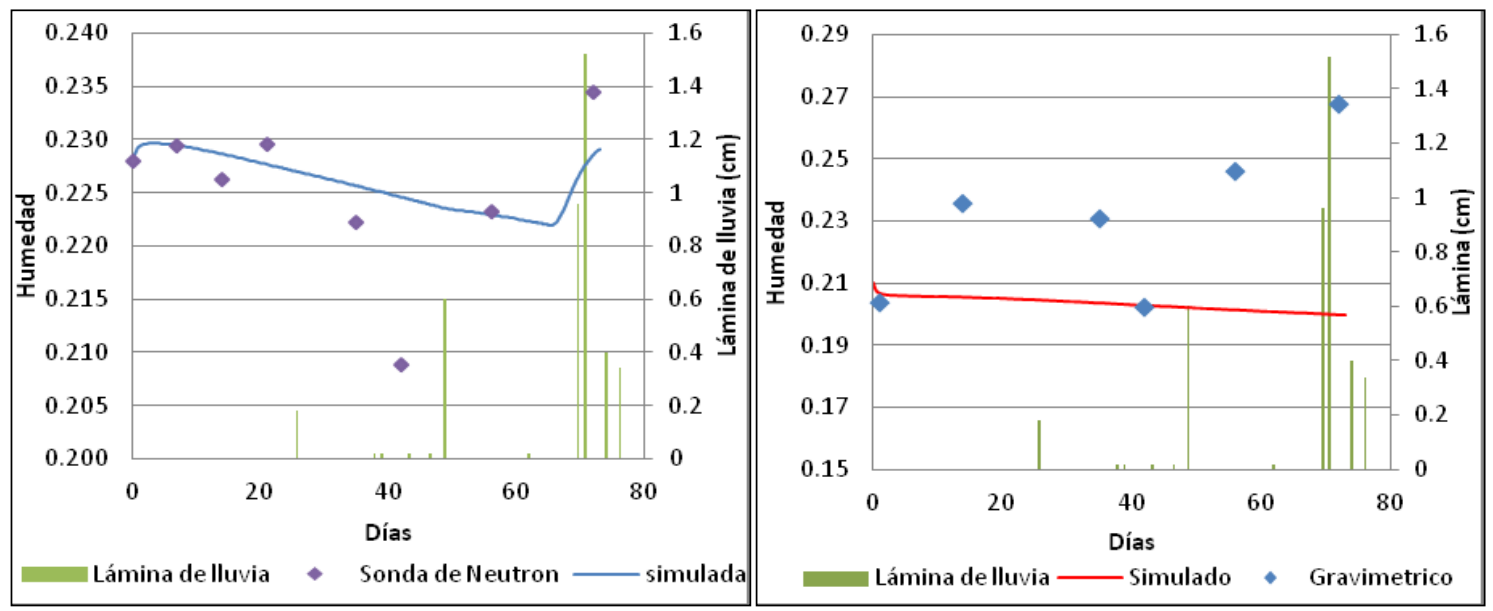

Figura 3. Resultados de la modelación con Hydrus a profundidad $90 \mathrm{~cm}$. Derecha: Simulación con el modelo ajustado para la sonda de neutrones. Izquierda: Simulación para el modelo ajustado con los datos del método gravimétrico.

De la Tabla 3 se observa que el valor del coeficiente de adsorción Kd del horizonte A y $\mathrm{AC}$ se encuentra dentro de los rangos publicados en la literatura, dentro de los valores de $\mathrm{Kd}$ estimados estos fueron los que mejor ajuste tuvieron con los datos obtenidos y están dentro los valores obtenidos de referencia $0.2-2 \mathrm{~L} / \mathrm{kg}$ y son aproximados a los determinados para los suelos de Córdoba (Hang y Sereno, 2002). 
Tabla 3. Valores obtenidos de la modelación inversa del escenario 1 y 2.

\begin{tabular}{cccccc}
\hline \multirow{2}{*}{ Horizonte } & \multicolumn{2}{c}{ escenario 1} & & \multicolumn{2}{c}{ escenario 2} \\
\cline { 2 - 3 } \cline { 6 - 6 } & $\mathrm{Kd}(\mathrm{L} / \mathrm{Kg})$ & S.E. & & $\mathrm{Kd}(\mathrm{L} / \mathrm{Kg})$ & S.E. \\
\hline $\mathrm{A}$ & 0.41 & 0.0191 & & 0.1647 & 0.015 \\
$\mathrm{AC}$ & 0.61 & 0.0277 & & 0.2026 & 0.24 \\
\hline
\end{tabular}

Los valores obtenidos de dispersión longitudinal presentan un intervalo de error muy amplio lo cual los hacen muy poco exactos para considerarlos. No obstante el valor obtenido para el ajuste de cada uno de los datos observados vs los simulados estimados por medio del error medio cuadrático $\mathrm{R}^{2}$ es de 0.9278 y 0.8772 el cual representa un buen ajuste del modelo de neutrones a los datos observados pero para el modelo gravimétrico los resultados no fueron tan buenos. Algunas de las causas que pueden modificar este coeficiente es la afectación por el \% de materia orgánica y el $\mathrm{pH}$, causando que para mayores $\mathrm{pH}$ y menor contenido de materia orgánica el coeficiente de adsorción disminuya.

\section{CONCLUSIONES}

El movimiento del agua en el suelo es el principal mecanismo para la transferencia de contaminantes a las aguas superficiales y subterráneas. La física del agua en el suelo y el movimiento de solutos pueden utilizarse para determinar el comportamiento de estos materiales. El movimiento de solutos a través de la zona no saturada, es particularmente importante en lo referente a la contaminación ambiental y agronómica. El transporte de solutos está afectado por procesos químicos y físicos de no-equilibrio.

Para ello y debido a la complejidad del problema y como se expresó en los puntos anteriores se hace necesario abordar y resolver distintos aspectos, por un lado el proceso de infiltración en suelo que requiere de la medición en campo de los procesos de infiltración frente a eventos de humedecimiento y secado y la determinación de funciones hidráulicas del suelo y por otro lado el abordaje de las mediciones en campo de los herbicidas y su posterior modelación matemática.

La concentración de plaguicidas en diferentes matrices ambientales se estima en base a muestreos basados en métodos estadísticos. En general, debido a la gran variabilidad espacial de las concentraciones de los distintos plaguicidas en el medio, los valores obtenidos en un muestreo sólo son una aproximación a la realidad. Por lo tanto, el mayor o menor grado de certeza en la obtención de los datos colectados y su interpretación depende en gran medida de un adecuado muestreo, de la recolección de la muestra y de la preservación de éstas.

Con relación a la aplicación de herbicidas la pulverización debería ser realizada bajo el concepto de aplicación de plaguicidas, definido como "el empleo de todos los conocimientos científicos necesarios para que un determinado fitoterápico llegue al blanco en cantidad suficiente para cumplir su cometido sin provocar contaminación ni derivas. Esta exigencia implica trabajar con un enfoque sistémico, que contemple el análisis y la interacción de múltiples variables. Una interpretación integrada del proceso de aplicación de plaguicidas permitirá la adecuada preparación de los pulverizadores para realizar una tarea exitosa.

De acuerdo con los resultados obtenidos, la simulación numérica resulta una herramienta válida para el estudio del movimiento del flujo y el transporte de sustancias contaminantes en la zona no saturada. En este sentido, el modelado ha permitido interpretar los resultados experimentales y establecer algunos comportamientos con respecto al flujo y el transporte de contaminantes por la columna de suelo simulada.

En atrazina se detectó un pico de concentración en todas las profundidades a los siete días después de la aplicación del herbicida al suelo. Dado que no se registró ningún pico 
similar dentro de las modelaciones se puede considerar la mayor parte del flujo de agua que produjo el transporte de atrazina haya sido por los macroporos del suelo o presencia de trazas de los cultivos anteriores.

Sin embargo, una limitada disponibilidad de datos obtenidos de estudios a campo representa la principal limitante para evaluar la validez de estos modelos, a fin de que puedan utilizarse con confianza para los propósitos para los que fueron creados.

A fin de minimizar el daño ambiental que produce la aplicación de los herbicidas su aplicación debe realizarse teniendo en cuenta, estrictamente, las condiciones de las plantas, el suelo y el ambiente, así como las dosis y los procedimientos de uso adecuados no siendo esto suficiente.

Finalmente y en relación a los mismos es importante destacar que en Argentina no existen valores de referencia sobre las concentraciones de pesticidas ni las profundidades que generan contaminación del suelo, ni en los metabolitos o productos químicos o microbianos.

\section{REFERENCIAS}

ARBAT, G.; PUIG, J.; BARRAGÁN, J.; BONANY, J.; RAMÍREZ DE CARTAGEN, F. Modelación directa e inversa de la dinámica del agua en una plantación de manzanos regada por micro aspersión. In: SAMPER CALVETE, F. J.; PAZ GONZÁLEZ, A. Estudios de la zona no saturada del suelo. 2005. Disponible en: http://www.zonanosaturada.com/publics/ZNS05/area_4/01.pdf. Acceso en: jul. 2014.

BEDMAR, F.; COSTA, J. L.; SUERO, E.; JIMÉNEZ, D. Transport of atrazine and metribuzin in three soils of the humid pampas of Argentina. Weed Technology, v. 18, p. 1-8, 2004. http://dx.doi.org/10.1614/WT-02-056

BRAY, R. H.; KURTZ, L. T. Determination of total, organic and available forms of phosphorus in soil. Soil Science, v. 59, p. 39-45, 1945.

CHAPMAN, D. Water quality assessments. London: Chapman \& Hall, 1992.

DÍAZ TRUJILLO, M. V. Estudios del sistema suelo-surfactante-plaguicida en los procesos se adsorción y desorción de atrazina, MBT y clorpirifos. 2007. 96f. Tesis (Maestría en Química) - Facultad de Ciencias Químicas y Farmacéuticas, Universidad de Chile, Santiago de Chile, 2007.

DOLL, J. Los herbicidas: modo de actuar y síntomas de toxicidad. Cali: Centro Internacional de Agricultura Tropical, 1982.

FEDDES, R.; KOWALIK, P.; ZARADNY, H. Simulation of filed water use and crop yield. Wageningen: Pudoc for the Centre for Agricultural Publishing and Documentation, 1978.

GARDNER, W. Water uptake and salt distribution patterns in saline soils. In: INTERNATIONAL ATOMIC ENERGY AGENCY (Ed.). Isotope ans radiation techniques in soil physics and irrigation studies. Vienna, 1967. p. 335-341.

GIULIANO, G. Groundwater vulnerability to pesticides: an overview of approaches and methods of evaluation. FUNARI, E.; VIGHI, M. (Eds.). Pesticide risk in groundwater Boca Raton: CRC Press, 1995. p. 101-118.

GOSS, D. W. Screening procedure for soils and pesticides for potential water quality impacts. Weed Technology, v. 6, p. 701-708, 1992. 
HANG, S.; SERENO, R. Adsorción de atrazina y su relación con las características sedimentológicas y el desarrollo del perfil de dos suelos de la Provincia de Córdoba. Revista de Investigación Agropecuaria, v. 31, n. 3, p. 73-87, 2002.

HORNSBY, A. Site-specific pesticide recommendations: the final step in environmental impact prevention. Weed Technology, v. 6, p. 736-742, 1992. http://www.jstor.org/stable/3987243

INTA MANFREDI, E. Caracterización del Área Bajo Riego suplementario en la Provincia de Córdoba. Córdoba: Manfredi, 2010.

KJELDAHL, J. A new method for the estimation of nitrogen in organic compounds. Analitical Chemistry, v. 22, p. 366, 1883.

MONTEITH, J. L. Evaporation and surface temperature. Quarterly Journal of Royal Meteorological Society, v. 107, p. 1-27, 1981. http://dx.doi.org/10.1002/ qj.49710745102

ROBINSON, D. A.; JONES, S. B.; WRAITH, M. J.; OR, D.; FRIEDMAN, S. P. A review of advances in dielectric and electrical conductivity measurement in soils using time domain reflectometry. Vadose Zone Journal, v. 2, p. 444-475, 2003. http://dx.doi.org/10.2136/vzj2003.4440

ROMERA PÉREZ, M. Agricultura ecológica. 2010. Disponible en: http://www.infoagro.com/agricultura_ecologica/agricultura_ecologica2.htm. Acceso en: 05 out. 2011.

ŠIMUNEK, J.; ŠEJNA, M.; VAN GENUCHTEN, M. TH. The DualPerm Module for HYDRUS (2D/3D) simulating two-dimensional water movement and solute transport in dual-permeability porous media, Version 1.0. Prague: PC Progress, 2012. $32 \mathrm{p}$.

VEREECKEN, H.; HUISMAN, J. A.; BOGENA, H.; VANDERBORGHT, J.; VRUGT, J. A.; HOPMANS, J. W. On the value of soil moisture measurements in vadose zone hydrology: a review. Water Resources Research, v. 44, n. 4, 2008. http://dx.doi.org/10.1029/2008WR006829

WAGENET, R. J.; RAO, P. S. C. Modeling pesticide fate in soils. In: CHENG, H. H. Pesticides in the soil environment: processes, impacts and modeling. Madison: SSSA Inc., 1990. p. 351-398.

WALKEY, A.; BLACK A. An examination of the Degtjareff method for determining soil organic matter, and proposed modification of the chromic acid titration method. Soil Science, v. 37, p. 29-38, 1934. 